\title{
Crystal Structure of 1-( $m$-Chlorophenyl)biguanide Hydrochloride
}

\author{
Gabriela LóPez-Olvera and Manuel Soriano-García ${ }^{\dagger}$ \\ Departamento de Bioquímica, Instituto de Química, UNAM, Circuito Exterior, \\ Ciudad Universitaria, Coyoacán, México D. F. 04510, México
}

\begin{abstract}
$\mathrm{C}_{8} \mathrm{H}_{11} \mathrm{Cl}_{2} \mathrm{~N}_{5}$ is monoclinic, $P 2_{1} / n$. The unit-cell dimensions at $293 \mathrm{~K}$ are $a=9.3569(9), b=13.6832(14), c=$ $18.4879(19) \AA, \beta=98.411(2)^{\circ}, V=2341.6(4) \AA^{3}, D_{\mathrm{x}}=1.408 \mathrm{~g} / \mathrm{cm}^{3}$, and $Z=8$. The $R$ value is 0.043 for 4129 reflections. The monoclinic unit cell contains two crystallographic independent molecules, A and $\mathrm{B}$. $\mathrm{The} \mathrm{Cl}(1)$ and $\mathrm{Cl}(4)$ ions are coordinated to 5 and $6 \mathrm{~N}$ atoms, respectively. In the crystal, there are two hydrogen bonds between N5A and N3B:N5B and N3A of the neighboring molecules, and constitute the major intermolecular attraction and packing force. These two molecules make a centro-symmetric dimer.
\end{abstract}

(Received June 7, 2004; Accepted August 16, 2004; Published on web November 4, 2004)

5-Hydroxytryptamine 3 (5- $\left.\mathrm{HT}_{3}\right)$ receptors are unique among serotonin receptors in that, rather than being coupled to $G$ proteins, they are ligand-gated ion channels. ${ }^{1}$ Allosteric or cooperative interactions may occur at $5-\mathrm{HT}_{3}$ receptors. These interactions have also been suggested based on preliminary

Table 1 Crystal and experimental data

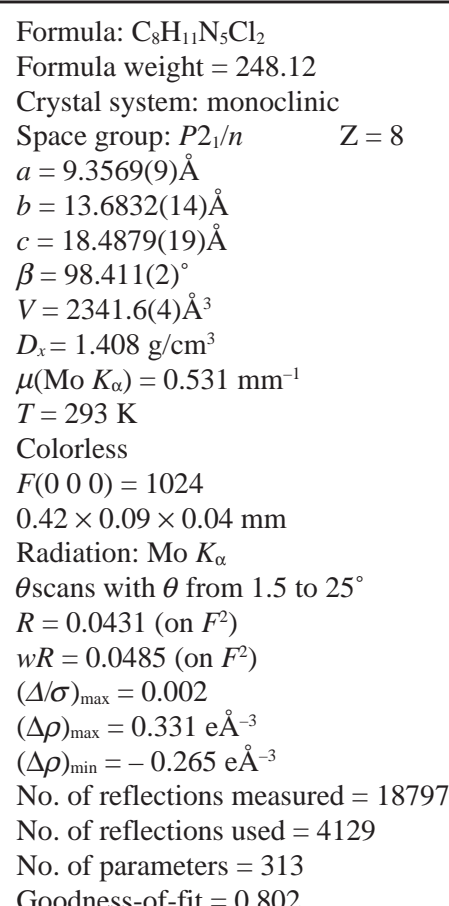

Goodness-of-fit $=0.802$

Measurement: Bruker Smart Apex CCD diffractometer

Program system: SMART V 5.625 and SAINT V 6.23C

Structure determination: direct methods (SHELXS-97)

Refinement: full matrix least-squares (SHELXL-97)

† To whom correspondence should be addressed.

E-mail: soriano@servidor.unam.mx studies using NG-108-15 cell membranes, which showed that the rate of dissociation of the $5-\mathrm{HT}_{3}$ receptor antagonist $\left[{ }^{3} \mathrm{H}\right] \mathrm{RS}$ 42358 depends upon the ligand used to prevent rebinding. ${ }^{2}$ To characterize further putative allosteric interactions at $5-\mathrm{HT}_{3}$ receptors, steady-state and kinetic radioligand binding studies have been conducted using the receptor agonist 1- $(m-$ chlorophenyl)biguanide. This suggests that the binding of this agonists to unoccupied sites on the receptor can increase the receptor's affinity for prebound ligands, and thereby slow their dissociation. ${ }^{3,4}$

In this paper, we describe an X-ray structure determination of 1-( $m$-chlorophenyl)biguanide hydrochloride. The crystals were prepared from a commercially available compound.

X-ray data for crystals of the title compound were collected by graphite-monochromatized Mo $K_{\alpha}$ radiation at $293 \mathrm{~K}$. No absorption correction was applied. The structure was solved by direct methods and refined by full-matrix least-squares with anisotropic temperature factors for the non-hydrogen atoms. In both molecules, the $\mathrm{C} 4, \mathrm{C} 5$ atoms of the phenyl rings show relatively high temperature factors, which may be due to a localized molecular disorder. The hydrogen atoms bonded to $\mathrm{C}$

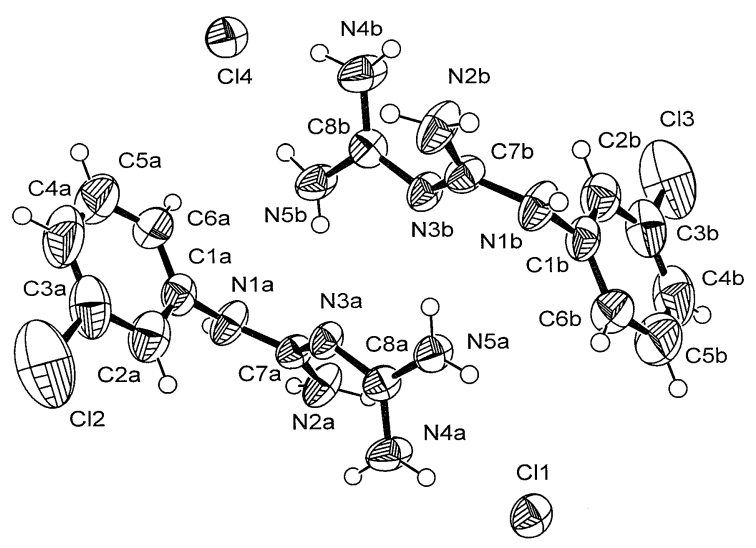

Fig. 1 Molecular structure of 1-( $m$-chlorophenyl)biguanide hydrochloride with atom labeling. Thermal ellipsoids are drawn at the $50 \%$ probability level. 
Table 2 Final atomic coordinates and equivalent isotropic temperature factors $\left(\AA^{2}\right)$

\begin{tabular}{|c|c|c|c|c|}
\hline Atom & $x$ & $y$ & $z$ & Ueq \\
\hline $\mathrm{Cll}$ & $0.04414(10)$ & $0.09903(5)$ & $0.08914(5)$ & $0.0531(3)$ \\
\hline $\mathrm{Cl} 2$ & $0.62450(15)$ & $0.58380(8)$ & $0.06880(8)$ & $0.1397(6)$ \\
\hline N1A & $0.7991(3)$ & $0.23582(18)$ & $0.13644(15)$ & $0.0554(9)$ \\
\hline $\mathrm{N} 2 \mathrm{~A}$ & $0.6957(3)$ & $0.0903(2)$ & $0.10399(18)$ & $0.0570(10)$ \\
\hline N3A & $0.5694(3)$ & $0.20863(16)$ & $0.16262(14)$ & $0.0433(8)$ \\
\hline $\mathrm{N} 4 \mathrm{~A}$ & $0.3871(3)$ & $0.1312(3)$ & $0.08038(18)$ & $0.0635(10)$ \\
\hline N5A & $0.3493(3)$ & $0.1725(2)$ & $0.19438(18)$ & $0.0509(9)$ \\
\hline $\mathrm{C} 1 \mathrm{~A}$ & $0.8034(4)$ & $0.3369(3)$ & $0.1522(2)$ & $0.0518(10)$ \\
\hline $\mathrm{C} 2 \mathrm{~A}$ & $0.7137(4)$ & $0.4021(3)$ & $0.1098(2)$ & $0.0677(12)$ \\
\hline $\mathrm{C} 3 \mathrm{~A}$ & $0.7325(5)$ & $0.5010(3)$ & $0.1232(3)$ & $0.0810(15)$ \\
\hline $\mathrm{C} 4 \mathrm{~A}$ & $0.8360(6)$ & $0.5350(3)$ & $0.1782(3)$ & $0.102(2)$ \\
\hline C5A & $0.9212(5)$ & $0.4698(3)$ & $0.2189(2)$ & $0.1017(18)$ \\
\hline C6A & $0.9065(4)$ & $0.3714(3)$ & $0.2069(2)$ & $0.0767(14)$ \\
\hline C7A & $0.6831(4)$ & $0.1773(2)$ & $0.13327(18)$ & $0.0430(9)$ \\
\hline $\mathrm{C} 8 \mathrm{~A}$ & $0.4369(4)$ & $0.1700(2)$ & $0.1449(2)$ & $0.0423(9)$ \\
\hline $\mathrm{Cl} 3$ & $0.38389(16)$ & $-0.10897(8)$ & $0.43580(8)$ & $0.1379(6)$ \\
\hline $\mathrm{Cl} 4$ & $0.95829(10)$ & $0.37591(5)$ & $0.41495(5)$ & $0.0544(3)$ \\
\hline N1B & $0.2028(3)$ & $0.23733(19)$ & $0.36821(17)$ & $0.0543(9)$ \\
\hline N2B & $0.3058(3)$ & $0.3827(2)$ & $0.4035(2)$ & $0.0601(11)$ \\
\hline N3B & $0.4318(3)$ & $0.26683(16)$ & $0.34204(14)$ & $0.0437(8)$ \\
\hline N4B & $0.6157(3)$ & $0.3394(3)$ & $0.42646(19)$ & $0.0647(11)$ \\
\hline N5B & $0.6529(3)$ & $0.3031(2)$ & $0.31167(17)$ & $0.0526(10)$ \\
\hline $\mathrm{C} 1 \mathrm{~B}$ & $0.2008(4)$ & $0.1357(3)$ & $0.3527(2)$ & $0.0516(10)$ \\
\hline $\mathrm{C} 2 \mathrm{~B}$ & $0.2913(4)$ & $0.0718(3)$ & $0.3951(2)$ & $0.0645(12)$ \\
\hline $\mathrm{C} 3 \mathrm{~B}$ & $0.2761(5)$ & $-0.0269(3)$ & $0.3814(3)$ & $0.0782(14)$ \\
\hline $\mathrm{C} 4 \mathrm{~B}$ & $0.1752(6)$ & $-0.0616(3)$ & $0.3269(3)$ & $0.0984(19)$ \\
\hline $\mathrm{C} 5 \mathrm{~B}$ & $0.0868(5)$ & $0.0020(3)$ & $0.2850(2)$ & $0.1000(17)$ \\
\hline $\mathrm{C} 6 \mathrm{~B}$ & $0.0987(4)$ & $0.1010(3)$ & $0.2973(2)$ & $0.0678(12)$ \\
\hline $\mathrm{C} 7 \mathrm{~B}$ & $0.3193(4)$ & $0.2961(2)$ & $0.37176(19)$ & $0.0436(9)$ \\
\hline $\mathrm{C} 8 \mathrm{~B}$ & $0.5630(4)$ & $0.3048(2)$ & $0.3610(2)$ & $0.0423(9)$ \\
\hline
\end{tabular}

$U_{\text {eq }}=(1 / 3) \sum_{i} \Sigma_{j} U_{i j}\left(a_{i}^{*} a_{j}^{*}\right)\left(\boldsymbol{a}_{i} \cdot \boldsymbol{a}_{j}\right)$.

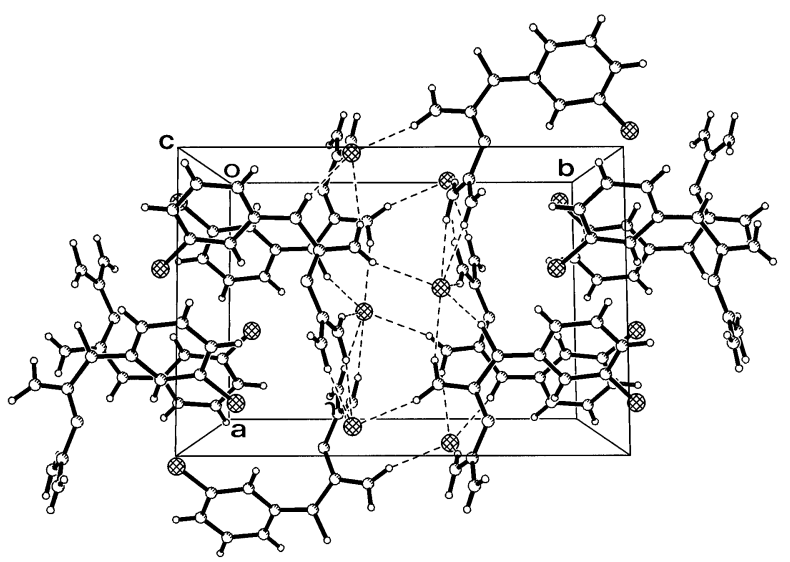

Fig. 2 Crystal-structure drawing. The broken lines show $\mathrm{Cl} \cdots \mathrm{H}$ interactions.

atoms were assigned based on the expected bonding geometry, and the $\mathrm{H}$ atoms bonded to $\mathrm{N}$ atoms were found in a differenceFourier map and refined. The software used to prepare the material for publication was PARST97.5 Table 1 summarizes the crystal and experimental data. The molecular structure is shown in Fig. 1. The bond lengths for non-H atoms are listed in Table 2.

The monoclinic unit cell contains two crystallographicindependent molecules, $\mathrm{A}$ and $\mathrm{B}$. The $\mathrm{Cl}(1)$ and $\mathrm{Cl}(4)$ ions are
Table 3 Bond distances $(\AA)$

\begin{tabular}{|c|c|c|c|}
\hline$C 2-C 3 A$ & $1.738(4)$ & $\mathrm{N} I \mathrm{~A}-\mathrm{C7A}$ & $1.343(4)$ \\
\hline N1A-C1A & $1.414(4)$ & $\mathrm{N} 2 \mathrm{~A}=\mathrm{C} 7 \mathrm{~A}$ & $1.320(4)$ \\
\hline N3A - C7A & $1.333(4)$ & $\mathrm{N} 3 \mathrm{~A}=\mathrm{Cs} \mathrm{A}$ & $1.344(4)$ \\
\hline N4A - CsA & $1.326(4)$ & $\mathrm{N} 5 \mathrm{~A}-\mathrm{C} 8 \mathrm{~A}$ & $1316(4)$ \\
\hline CIA - C6A & $1.375(5)$ & $\mathrm{C} 1 \mathrm{~A}-\mathrm{C} 2 \mathrm{~A}$ & $1.385(4)$ \\
\hline $\mathrm{C} 2 \mathrm{~A}-\mathrm{C} 3 \mathrm{~A}$ & $1.384(4)$ & $C 3 A-C 4 A$ & $1372(5)$ \\
\hline $\mathrm{C} 4 \mathrm{~A}-\mathrm{C} 5 \mathrm{~A}$ & $1.351(5)$ & $\operatorname{c5} A-\operatorname{Cos} A$ & $136 \%(4)$ \\
\hline $\mathrm{C} 13-\mathrm{C} 3 \mathrm{~B}$ & $1.730(4)$ & $\mathrm{N} 1 \mathrm{~B}=\mathrm{C} 7 \mathrm{~B}$ & $1349(4)$ \\
\hline $\mathrm{N} 1 \mathrm{~B}=\mathrm{C} 1 \mathrm{~B}$ & $1.420(4)$ & $\mathrm{N} 2 \mathrm{~B}-\mathrm{C} 7 \mathrm{~B}$ & $1337(4)$ \\
\hline $\mathrm{N} 3 \mathrm{~B}-\mathrm{C} 7 \mathrm{~B}$ & $1.319(4)$ & N3B - C8B & $1332(4)$ \\
\hline $\mathrm{N} 4 \mathrm{~B}-\mathrm{CKB}$ & $1.525(4)$ & N5B-C8B & $1.32 \times(4)$ \\
\hline $\mathrm{C} 1 \mathrm{~B}=\mathrm{C} 2 \mathrm{~B}$ & $1.379(4)$ & CIB - C6B & $1.379(4)$ \\
\hline $\mathrm{C} 2 \mathrm{~B}-\mathrm{C} 3 \mathrm{~B}$ & $1.377(4)$ & $\mathrm{C} 3 \mathrm{~B}-\mathrm{C} 4 \mathrm{~B}$ & $1.363(5)$ \\
\hline CAB - CSB & $1.362(5)$ & C5B- C6B & $1.374(4)$ \\
\hline
\end{tabular}

coordinated to the 5 and $6 \mathrm{~N}$ atoms, respectively. The mean $\mathrm{Cl}^{-}$ to $\mathrm{N}$ distance is $3.264(4) \AA$, which is slightly different compared with $3.272(2) \AA$ in the 1-(p-chlorophenyl)biguanide hydrochloride. ${ }^{6}$ There is a close resemblance between the dimensions of the biguanide residues in this structure and in 1( $p$-chlorophenyl)biguanide hydrochloride. ${ }^{6}$ Figure 2 shows the packing of the molecules in the unit cell. In the crystal, there are two hydrogen bonds between N5A and N3B and N5B and $\mathrm{N} 3 \mathrm{~A}$ of neighboring molecules, which constitute the major intermolecular attraction and packing force. These two molecules make a centro-symmetric dimer. The N5A $\cdots \mathrm{N} 3 \mathrm{~B}$, $\mathrm{H} 5 \cdots \mathrm{N} 3 \mathrm{~B}, \quad \mathrm{~N} 5 \mathrm{~B} \cdots \mathrm{N} 3 \mathrm{~A}, \quad \mathrm{H} 18 \cdots \mathrm{N} 3 \mathrm{~A}$ distances are 3.016(4), $2.17(3), 3.041(4), 2.26(3) \AA$ and the N5A-H5 $\cdots$ N3B, N5BH18 $\cdots$ N3A angles are $172(3)$ and $174(3)^{\circ}$, respectively. There are several $\mathrm{N} \cdots \mathrm{Cl}$ intermolecular interactions that stabilize the molecules in the crystal, such as: $\mathrm{N} 2 \mathrm{~A} \cdots \mathrm{Cl} 1(x+1,+y,+z)$ 3.314(3) $\AA$; N2A $\cdots \mathrm{Cl} 4(-x+3 / 2,+y-1 / 2,-z+1 / 2) 3.264(3) \AA$; N4A $\cdots \mathrm{Cl} 4(x-1 / 2,-y+1 / 2,+z-1 / 2) 3.225(4) \AA$; $2 \mathrm{~B} \cdots \mathrm{Cl} 4(x-$ $1,+y,+z) 3.291(3) \AA$; N1B $\cdots \mathrm{Cl} 4(x-1,+y,+z) 3.187(3) \AA$; $\mathrm{N} 2 \mathrm{~B} \cdots \mathrm{Cl} 1(-x+1 / 2,+y+1 / 2,-z+1 / 2) 3.271(3) \AA$, and N4B $\cdots$ Cl1 $(x+1 / 2,-y+1 / 2,+z+1 / 2) 3.285(4) \AA$.

\section{Acknowledgements}

Gabriela López Olvera give thanks to the Sistema Nacional de Investigadores, SEP, MEXICO for her financial support during her stay at the Chemistry Institute, UNAM

\section{References}

1. J. A. Peters, H. M. Malone, and J. J. Lambert, Tre. Pharmacol. Sci., 1992, 13, 391.

2. D. W. Bonhaus, R. M. Eglen, and E. H. F. Wong, Soc. Neurosci. Abstr., 1992, 18, 1518.

3. G. J. Kilpatrick, A. Butler, J. Burride, and A. W. Oxford, Eur. J. Pharmacol., 1993, 182, 193.

4. D. W. Bonhaus, E. Stefanich, D. N. Loury, S. A. O. Hsu, R. M. Eglen, and E. H. F. Wong, J. Neurochem., 1995, 65, 104.

5. M. Nardelli, J. Appl. Cryst., 1995, 28, 659.

6. C. J. Brown, and L. Sengier, Acta Cryst., 1984, C40, 1294 